\author{
Bilal Ahmed ${ }^{\star}$ \\ Department of Prosthodontics, Army Medical \\ College, National University of Sciences of \\ Technology (Nust) Islamabad \\ Dates: Received: 22 July, 2015; Accepted: 30 July, \\ 2015; Published: 31 July, 2015 \\ ${ }^{*}$ Corresponding author: Dr. Bilal Ahmed, \\ Associate Professor, Department of Prosthodontics, \\ Army Medical College, Abid Majeed Road, \\ Rawalpindi Cantt, Tel: +923216008263; E-mail: \\ drbilalahmed79@hotmail.com; drbilalahmed79@ \\ amcollege.nust.edu.pk \\ www.peertechz.com \\ ISSN: 2455-4634
}

Keywords: Prosthodontic rehabilitation; Soft palate Obturator

\section{Case Report \\ Rehabilitation of Surgically \\ Resected Soft Palate with Interim Velopharyngeal Obturator}

\section{Introduction}

The morphology of jaws are made in such way that they aid in both function and aesthetics of a person. The main function of the soft palate is that it separates the nasal and oral part of pharynx that in turn aids in speech and swallowing. The velophayngeal (VP) valve consists of soft palate, lateral walls of pharynx and posterior walls of pharynx. During speech, the soft palate is raised that controls the airflow through the nose and mouth and lateral and posterior walls of pharynx constricts to produce speech [1]. Variation in the contribution of each of these components produces several patterns of sphincteric closure or attempted closure of the VP portal that were described by Skolnick et al. [2]. Adequate VP closure is required during swallowing and production of all consonants except for the nasal ones [3].

VP impairment can be due to inadequacy and insufficiency. VP inadequacy is caused by neuromuscular disorder of an anatomically intact VP sphincter [4]

VP insufficiency can be either congenital as in cleft palate cases, or acquired as in surgical removal of a part of palate due to palatal carcinoma resection [4] or due to some infections. Malignant tumors of the hard palate account for 1-5\% of the tumors of the oral cavity. Adenoid cystic carcinoma being one of the malignant tumors of oral cavity is a salivary gland tumor which most commonly occurs in the minor salivary glands [5]. It most commonly occurs in males than in females [5]. Most of these tumors are diagnosed late and the final option left is aggressive surgical resection [5]. This treatment accounts for velopharyngeal insufficiency resulting in nasal regurgitation of liquids, hyper nasal speech, nasal escape, disarticulations and impaired speech intelligibility. In addition normal mechanism of swallowing is also disturbed [6-8].

A soft palate or velopharyngeal obturator (speech and feeding aid device) replaces the missing part of muscles, fills the defects and either can be a metal framework or all plastic (acrylic) prosthesis [9]. In each case the patient will require some functional adjustments to the prosthesis as this is an area that moves every time the patient speaks or swallows. Adjustment of these prostheses is a fine balance between swallowing and speaking. If the valve is closed too tightly then swallowing is very good but speaking will sound as if the patient has a cold or stuffy nose (hypo nasality). If the valve is left more open then speech will sound better but food/liquid leakage when swallowing may be increased. In addition, healing from surgery or radiation treatment will alter this area and require frequent adjustments until all healing is completed [9].

These obturators can have mobile distal extensions or bulbs with a flexibility to have synchronized movements during functional opening and closure of the adjacent soft tissues around a customized hinged joint. However, fixed or stable distal extensions with balanced functional adjustments are more recommended as these are less complicated, easily fabricated and adequately functional for successful outcomes. Caring for the prosthesis is relatively easy. Simply brushing the prosthesis or rinsing with soap and water when cleaning the teeth is adequate. Effervescent partial denture cleaning tablets can be used as well when the prosthesis is soaking while the patient is sleeping. These prostheses aid in improving a complex muscular valve dysfunction. With patience and time these prosthetic devices can be fine-tuned to be very functional and comfortable [9].

\section{Case Report}

A 23 Year old female was referred from Department of Oral and Maxillofacial Surgery with a history of surgical resection of soft palate area three months earlier due to a sudden development of nodular mass which was later diagnosed to be Adenoid cystic cell carcinoma of soft palate. The patient was followed up for any recurrence or swelling and later sent for rehabilitation of the lost intra oral structures as her major complaint was difficulty in swallowing, nasal regurgitation of food and intelligible hyper nasal voice. Otherwise, she had no pain or 
swelling. On examination, patient was found to have a big defect in the soft palate area up to the nasal septum. There was no uvula present and no velopharyngeal closure was possible. The dental status was intact with no missing teeth (Figure 1).

After detailed discussion and explanation to the patient, a palatal obturator was planned. The defect was recorded in alginate impression material and full extension palatal plate was fabricated in acrylic resin with wrought wire clasps on teeth \# 16, 23 and 26. The intaglio surface of the prosthesis was made very smooth and polished to prevent entrapment of nasal discharge or food particles. The distal extension of the prosthesis was made fixed, stable and modified functionally until the patient was comfortable on deglutition (Figures $2,3)$.

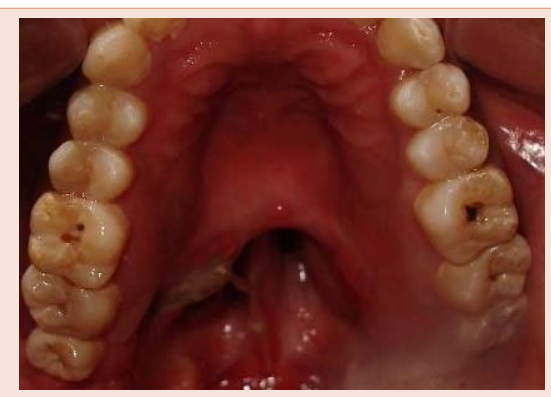

Figure 1: Intra oral view of resected soft palate.

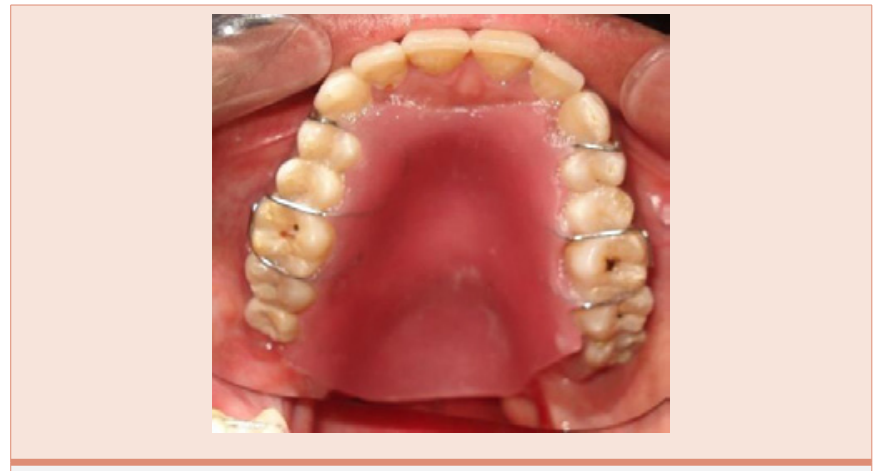

Figure 2: Obturator prosthesis in place.

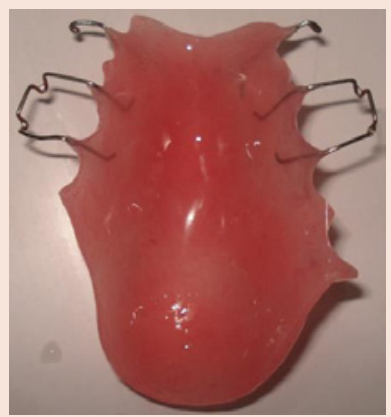

Figure 3: Intaglio surface of Obturator prosthesis.
The patient was followed up at 24 hours interval and was educated regarding the use, insertion removal protocol and oral hygiene maintenance. The patient was regularly followed up on monthly basis for one year and was found to be very comfortable and satisfied with the immediate improvement of phonetics and regurgitation without any aggressive restorative or surgical procedure.

\section{Discussion}

A pharyngeal obturator is a removable maxillary prosthesis which has a posterior extension that separates the oropharynx from nasopharynx [3]. This obturator prosthesis restores the defects of the soft palate and allows adequate closure of velopharyngeal sphincter [3]. Obturator provides the necessary barrier between nasopharynx and oropharynx which helps a patient during speech without letting excessive air to escape from nose along with restoring normal deglutition. The pharyngeal obturator consisted of a palatal portion, velar portion, pharyngeal portion (bulb), completing the velopharyngeal sphincter.

In an acquired palatal defect, especially a defect after tumor resection, obturator prosthesis is chosen over surgical repair for several reasons. One of the reason being that that the obturator allows easier inspection of the defect after surgical resection and the second reason that the patient might need adjunctive radiotherapy and thirdly that there's a slight chance of recurrence of the carcinoma [7]. In order for an obturator to work properly it should have an adequate retention and stability. In dentate patients retention is easily achieved with the help of existing teeth [3]. Prosthetic management with an obturator requires close cooperation between the prosthodontist and a phonetician (speech pathologist) [6].

Velopharyngeal insufficiency can result in perplexing problems as described earlier. The main use of an obturator is to minimize the resulting effects of a VP insufficiency i.e. to improve speech and swallowing without nasal leakage. Pharyngeal obturator/ speech bulb is a simple extension of acrylic from the oral part of the prosthesis. The extension should be up to the passavants ridge. Passavants ridge is a slight bulge on the posterior wall of the pharynx.

Rigid posterior extension of acrylic, as compared to the introduction of hinge joint between the oral and the pharyngeal part of the prosthesis as used by Ram was fabricated [4]. Rigid extension of acrylic provides better results compared to the hinge joint and patient discomfort is to a minimum [4]. The retention was achieved by wrought wire clasping of the existing dentition as discussed by Tuna et al. [3]. Extension of acrylic up to the passavants ridge can cause slight irritation. The irritating area can be marked and modifications are carried out to the comfort of the patient as was done for this patient.

Assessment of the obturator is important. Speech bulb or pharyngeal obturator may correct the nasal emission and food regurgitation but articulatory errors may persist. This is the reason why a speech evaluation after prosthetic rehabilitation is necessary. Articulation errors and nasal resonance during speech is evaluated by a speech pathologist and it is of utmost importance for optimizing results [9]. 
In this case report we were successfully able to rehabilitate the patient with a pharyngeal obturator within only a few days of treatment duration, restoring normal functions of speech and deglutition without any aggressive surgical therapy. This Pharyngeal obturator not only helped to restore normal function but also helped the patient psychosocially and as for other maxillofacial, prosthodontics appliances lead to improve the oral health related quality of life $[10,11]$.

\section{References}

1. Woo AS (2012) Velopharyngeal dysfunction, Semin Plast Surg 26: 170-177.

2. Skolnick ML, Mc CG, Barnes M (1973) The sphincteric mechanism of velopharyngeal closure. Cleft Palate J 286-305.

3. Tuna SH, Pekkan G, Gumus HO, Aktas A (2010) Prosthetic rehabilitation of velopharyngeal insufficiency: pharyngeal obturator prostheses with different retention mechanisms. Euro J Dent 4: 81-87.

4. Ram HK, Shah RJ (2013) A novel approach for Velopharyngeal prosthetic rehabilitation: Case series. International $\mathrm{J}$ of Healthcare \& Biomedical Research1: 70-76.

5. Lawal AO, Adisa AO, Kolude B, Adeyemi BF, Olajide MA (2013) A review of
413 salivary gland tumors in the head and neck region. J Clin Exp Dent 5: e218-222.

6. Tirelli G, Rizzo R, Biasotto M, Di Lenarda R, Argenti B, Gatto A, et al. (2010) Obturator prostheses following palatal resection: clinical cases. Acta Otorhinolaryngol Ital 30: 33-39.

7. Aboloyoun Al, Ghorab S, Farooq MU (2013) Palatal lifting prosthesis and velopharyngeal insufficiency: preliminary report. Acta Med Acad 42: 55-60.

8. Lin FH, Wang TC (2011) Prosthodontic rehabilitation for edentulous patients with palatal defect: report of two cases. J Formos Med Assoc 110:120-124.

9. (2005) The Glossary of Prosthodontic Terms, 8th ed. J Pros Dent 94: 10-92.

10. Lima-Gregio AM, Marino VC, Pegoraro-Krook MI, Barbosa PA, Aferri HC, et al. (2011) Nasalance and nasality at experimental velopharyngeal openings in palatal prosthesis: a case study. J Appl Oral Sci 19: 616-622.

11. Mahmood A, Ahmed B, Parveen N, Yazdanie N (2009) Oral health related quality of life in complete denture prosthodontics. Pak Oral Dent J 29: 397402.

12. Ahmed B, Hussain M, Mahmood A, Yazdanie N (2011) Maxillofacial rehabilitation of a large intra oral defect using fixed removable prosthesis. J Coll Physician Surg Pak 21: 52-54. 